\title{
Delayed Auditory Evoked Responses in Autism Spectrum Disorder across the Life Span
}

\author{
Junko Matsuzaki ${ }^{a}$ Matthew Ku ${ }^{a}$ Marissa Dipiero ${ }^{a}$ Taylor Chiang ${ }^{a}$ Joni Saby ${ }^{a}$ \\ Lisa Blaskey ${ }^{a}$ b Emily S. Kuschner ${ }^{a, b}$ Mina Kim $^{a}$ Jeffrey I. Berman ${ }^{a} \quad$ Luke Bloy $^{a}$ \\ Yu-han Chen ${ }^{a}$ John Dell ${ }^{a}$ Song Liu ${ }^{a}$ Edward S. Brodkin ${ }^{c}$ David Embick $^{d}$ \\ Timothy P.L. Roberts ${ }^{a, d}$ \\ a Lurie Family Foundations MEG Imaging Center, Department of Radiology, Children's Hospital of Philadelphia, \\ Philadelphia, PA, USA; ${ }^{\circ}$ Center for Autism Research, Children's Hospital of Philadelphia, Philadelphia, PA, USA; \\ 'Department of Psychiatry, Perelman School of Medicine at the University of Pennsylvania, Philadelphia, PA, USA; \\ ${ }^{\mathrm{d}}$ Department of Linguistics, University of Pennsylvania, Philadelphia, PA, USA
}

\section{Keywords}

Autism spectrum disorder · Magnetoencephalography · M50 $\cdot$ M100 $\cdot$ Tones $\cdot$ Language ability $\cdot$ Life span

\begin{abstract}
The M50 and M100 auditory evoked responses reflect early auditory processes in the primary/secondary auditory cortex. Although previous M50 and M100 studies have been conducted on individuals with autism spectrum disorder (ASD) and indicate disruption of encoding simple sensory information, analogous investigations of the neural correlates of auditory processing through development from children into adults are very limited. Magnetoencephalography was used to record signals arising from the left and right superior temporal gyrus during auditory presentation of tones to children/adolescents and adults with ASD as well as typically developing (TD) controls. One hundred and thirty-two participants (aged 6-42 years) were included into the final analyses (children/adolescents: TD, $n=36,9.21 \pm 1.6$ years; ASD, $n=58,10.07 \pm 2.38$ years; adults: TD $, n=19,26.97 \pm 1.29$ years; ASD, $n=19,23.80 \pm 6.26$ years). There were main effects of group on M50 and M100 latency ( $p<0.001)$ over
\end{abstract}

hemisphere and frequency. Delayed M50 and M100 latencies were found in participants with ASD compared to the TD group, and earlier M50 and M100 latencies were associated with increased age. Furthermore, there was a statistically significant association between language ability and both M50 and M100 latencies. Importantly, differences in M50 and M100 latencies between TD and ASD cohorts, often reported in children, persisted into adulthood, with no evidence supporting latency convergence.

๑) 2020 S. Karger AG, Basel

\section{Introduction}

Autism spectrum disorder ${ }^{1}$ (ASD) is a neurodevelopmental disorder characterized by impaired social and communication skills, and by repetitive and stereotyped behavior [1, 2]. Previous magnetoencephalographic

1 Individuals on the autism spectrum, their parents, and professionals in the field have unique and overlapping opinions regarding the use of person first (e.g., children with ASD) or identity first (e.g., autistic child) language (Kenny et al. [1]). With respect for divided opinions, we use both approaches to terminology in this paper.

\section{KARGER}

(c) 2020 S. Karger AG, Basel

karger@karger.com

www.karger.com/dne
Timothy P.L. Roberts, PhD

Children's Hospital of Philadelphia

3401 Civic Center Blvd

Philadelphia, PA 19104 (USA)

E-Mail ROBERTSTIM@email.chop.edu 
(MEG) studies have shown abnormal auditory cortical responses in the superior temporal gyrus (STG), such as delayed auditory response components in ASD cohorts compared to typically developing (TD) peers [3-16]. In particular, delayed responses have been observed for both auditory response components around $50 \mathrm{~ms}$ (M50) and $100 \mathrm{~ms}$ (M100), the responses primarily produced by neural activity from primary/association auditory cortex $[3,4,7]$. The M50 is analogous to the $\mathrm{Pa} / \mathrm{P} 1$ complex of the electroencephalographic (EEG) evoked responses; the M100 magnetic field is comparable to the N1 [5].

In an early MEG study, Gage et al. [3] reported delayed M100 latencies to sinusoidal tones in children with ASD (aged 8-16 years) and suggested altered auditory system development in ASD children, which may reflect abnormalities in cortical maturational processes in this population. Roberts et al. [7] also reported delayed M100 latencies in children with ASD compared to TD, suggesting disruption of simple sensory information encoding. Of note, Oram Cardy et al. [5] showed that while both M50 and M100 latencies were prolonged with respect to adult values, the M50 peak was prominent over the M100 peak in younger children $(<\sim 12$ years), which suggests this as a better probe to characterize development. Roberts et al. [8] confirmed M50 delays in ASD compared to TD, but diffusion tensor imaging findings implicating maturational changes in auditory pathway white matter as influencing conduction velocity in TD children [17] were not replicated in the cohort of children with ASD, leading to the hypothesis that additional mechanisms, for example, synaptic transmission, may also influence auditory latency delay [8].

Thus, although a number of studies have revealed delayed early auditory responses in children with ASD, it appears the maturational underpinnings of both response development and the mechanism underlying the observed delay in ASD are unclear. Furthermore, there are little data on MEG response delays in adults with ASD, raising the question of whether latencies in ASD and TD converge by adulthood, or whether delays evident in childhood persist through the life span. In light of the different mechanisms (i.e., degrees of association with white matter maturation) underlying response latency in TD versus ASD, either hypothesis remains plausible. To better understand neural correlates of auditory processing in ASD across the life span, the present study used MEG to measure cortical responses to auditory tone stimuli with frequencies (200, 300,500 , and $1,000 \mathrm{~Hz}$ ) identical to those used in the study by Roberts et al. [7] and compared them with responses from a TD cohort with similar age range. Although both the latency and amplitude of early auditory responses have been examined, the present study focuses on latency, since Roberts et al. [7] found statistically significant group differences in latency but not in amplitude.

Hypotheses were: (1) M50 and M100 latencies would be delayed in ASD and show maturational shortening with increasing age, and (2) latency delay would be associated with language aptitude. Finally, (3) we addressed the question as to whether M50 and M100 latency differences, previously reported between children with TD versus ASD, (a) are replicated in this study and (b) persist into adulthood, or whether there is ultimately latency convergence.

\section{Materials and Methods}

\section{Participants}

All participants were recruited from the Children's Hospital of Philadelphia (CHOP)/the University of Pennsylvania. Clinical and diagnostic testing was performed to confirm ASD diagnosis, administer cognitive assessments, and ensure that all participants met study inclusion but not exclusion criteria. Clinical assessments were performed by a licensed psychologist with expertise in ASD.

Children/Adolescents. The current sample represents children who participated in our research studies between 2008 and 2019. However, M50 and M100 data from these particular individuals have not been reported previously. Children with ASD had a prior diagnosis, typically made by an expert clinician in the CHOP Regional Autism Center or, more rarely, by community providers. Given the extensive clinical evaluations upon which original ASD diagnosis was made, an abbreviated diagnostic battery was used to confirm the original ASD diagnosis. The abbreviated battery included the Autism Diagnostic Observation Schedule (ADOS/ADOS-2) [18, 19], Social Communication Questionnaire (SCQ) [20] and Social Responsiveness Scale, 1st or 2nd Edition (SRS/SRS-2) [21, 22]. The Autism Diagnostic Interview-Revised (ADI-R) [23] was administered to parents for any participants who entered the study without a formal ASD diagnosis made by an expert clinician (e.g., ASD educational classification only) and for any child with a prior ASD diagnosis for whom a diagnostic discordance existed during the evaluation (e.g., a child who exceeded ADOS/ADOS-2 diagnostic cutoffs but was below SCQ cutoff). Cognitive ability was characterized with the Wechsler Intelligence Scale - Fourth Edition (WISC-IV) [24]. To rule out global cognitive delay, all subjects scored at or above the 2 nd percentile (SS $>70$ ) on at least one index of verbal (VIQ) or nonverbal (NVIQ) intellectual functioning. Estimated Full Scale IQ (FSIQ) was obtained from the WISC General Ability Index (GAI).

Adults. Adult participants with ASD were recruited from the Adult Autism Spectrum Program in the Department of Psychiatry, Perelman School of Medicine, at the University of Pennsylvania, as well as from cohorts of participants participating in prior MEG studies by the current investigators and prior studies at the CHOP Center for Autism Research. All adult ASD participants had a prior ASD diagnosis, made by an expert clinician according to DSM criteria. Diagnosis was confirmed at the time of participation by exceeding established cutoffs for the ADOS-2 as well as for either the SCQ (Lifetime; 20) or SRS-2 Adult-Informant Report [21, 22]. Individuals for whom informant report was not available were included in the ASD 
group if they had a documented prior diagnosis of ASD and exceeded established cutoffs on the ADOS-2 as well as on both the SRS-2 Adult-Self Report and Broad Autism Phenotype Questionnaire [25]. Individuals 1 point below diagnostic cutoffs on the ADOS- 2 were included if they exceeded cutoffs on 2 informant report questionnaires or on the ADI-R. Intellectual functioning was indexed with the Wechsler Abbreviated Scale of Intelligence-II (WASI-II) [26] and included estimates of both nonverbal (Perceptual Reasoning Index [PRI]) and verbal (Verbal Comprehension Index [VCI]) intellectual abilities. Inclusion criteria for the TD adults were scoring below the cutoff for ASD on all domains of the ADOS-2 and below cutoffs on informant and self-report questionnaires, along with performance above the 16th percentile on the Clinical Evaluation of Language Fundamentals - Fourth Edition (CELF-4 [27]; if within age range for this measure), WASI-II Verbal Comprehension Index, and an average of the Peabody Picture Vocabulary Test-4 (PPVT-4) [28] and Expressive Vocabulary Test-2 (EVT-2) [29].

The following inclusion/exclusion criteria were used for all participants: (1) no history of traumatic brain injury or other significant medical or neurological abnormality, or other genetic syndrome, (2) no active psychosis, (3) no MRI contraindications, (4) no significant sensory impairments (e.g., vision or hearing impairment), (5) English as a first language, and (6) no known drug or alcohol use prior to any study procedure.

The study was approved by the CHOP Institutional Review Board, and all participants' and/or their caregivers gave written informed consent. As indicated by institutional policy, where competent to do so, children over the age of 7 additionally gave verbal assent, in accordance with the principles of the Declaration of Helsinki.

\section{Auditory Stimuli}

Auditory stimuli were delivered via a sound pressure transducer and sound conduction tubing to the subject's peripheral auditory canal via ear tip inserts (ER3A; Etymotic Research, Elk Grove Village, IL, USA). Prior to the MEG recording, each participant's hearing threshold was determined, and the auditory stimuli were presented $45 \mathrm{~dB}$ SPL above threshold. The auditory stimuli consisted of $200,300,500$, and $1,000 \mathrm{~Hz}$ sinusoidal tones of $300-\mathrm{ms}$ duration. The tones were binaurally presented (digitized at 44,100 $\mathrm{Hz}$ with a 10 -ms rise time) in a random order with a 1-s interstimulus interval (jittered $100 \mathrm{~ms}$ ). One hundred and five tones at each of the 4 frequencies were presented for a total recording duration of approximately $10 \mathrm{~min}$.

\section{MEG Recording}

MEG data were obtained in a magnetically shielded room using a 275-channel whole-cortex CTF magnetometer (CTF MEG, Coquitlam, BC, Canada). At the start of the session, 3 head position indicator coils were attached to the scalp to provide continuous specification of the position and orientation of the MEG sensors relative to the head [7]. Foam wedges were inserted between the side of each participant's head and the inside of the MEG dewar to increase participant comfort and ensure that the head remained in the same place in the dewar across recording sessions. To minimize fatigue and encourage an awake state, subjects viewed a silent movie projected on to a screen positioned at a comfortable viewing distance. To aid in the identification of eyeblink activity, electrooculogram (EOG, bipolar oblique, and upper right and lower left sites) was collected. Electrodes were also attached to the left and right collarbone for electrocardiogram (ECG) recording.

Delayed Auditory Response in ASD

across the Life Span

\section{Data Analysis}

All analyses were performed blind to the participant group. Epochs $100 \mathrm{~ms}$ prestimulus to $500 \mathrm{~ms}$ poststimulus were defined from the continuous recording. To correct for eyeblinks, a typical eyeblink was manually identified in the raw data (including EOG) for each participant. The pattern search function in BESA Research 6.1 (BESA GmbH, Germany) scanned the raw data to identify other blinks and computed an eyeblink average. An eyeblink was modeled by its first component topography from principal component analysis, typically accounting for $>99 \%$ of the variance in the eyeblink average. In addition to eyeblink activity, a heartbeat average was obtained, and heartbeat activity was modeled by the first 2 principal component topographies of a heartbeat average, typically accounting for $>85 \%$ of the variance in the heartbeat average. Scanning the eyeblink- and heartbeat-corrected raw data, epochs with artifacts other than blinks and heartbeats were rejected by amplitude and gradient criteria (amplitude $>300 \mathrm{fT}$, gradients $>25 \mathrm{fT} /$ sample). Noncontaminated epochs were averaged, and a 1 - (12 dB/octave, zero phase) to $55-\mathrm{Hz}$ ( $48 \mathrm{~dB} /$ octave, zero phase) band pass filter was applied. Using all 275 channels of MEG data, determination of the strength and latency of M50 and M100 sources in the left and right STG was accomplished by applying a standard source model to transform each individual's raw MEG surface activity into brain space (MEG data co-registered to each individual's anatomic MRI) using a model with multiple sources [30-32]. In particular, the standard source model applied to each subject was constructed by including (1) left and right STG dipole sources (anatomically placed at left and right Heschl's gyrus). The eyeblink and heartbeat source vectors derived for each participant were also included in each participant's source model to remove eyeblink and heartbeat activity $[33,34]$. The final source model served as a source montage for the raw MEG $[35,36]$. As such, the MEG sensor data were transformed from channel space into brain source space where the visualized waveforms were the modeled source activities. This spatial filter disentangled the source activities of the different brain regions that overlapped at the sensor level. Of note, although the latency of the 50- and 100-ms STG responses were obtained using a dipole source placed at a standard location, in each subject left (LH) and right hemisphere (RH), dipoles were oriented at the maximum of the individual M50 and M100. As such, orientation of the standard STG sources was optimized in each subject. Left and right M50 (50-125 ms) and M100 (100-250 ms) peaks were defined from the source waveform.

\section{Statistics}

Potential differences between groups (TD and ASD) in age and neuropsychological assessments were evaluated with independent sample $t$ tests. Potential effects of group (TD and ASD), frequency $(200,300,500$, and $1,000 \mathrm{~Hz})$, and hemisphere ( $\mathrm{LH}$ and $\mathrm{RH})$ on M50 and M100 latency were evaluated with full factorial linear mixed models (LMMs) using these factors as fixed effects, age as a covariate, and subject as a random effect. Hierarchical regression assessed associations of language aptitude (VIQ) and social impairment (SRS-2) on M50 and M100 latency beyond influences of age, group (TD and ASD), frequency $(200,300,500$, and 1,000 Hz), and hemisphere (LH and $\mathrm{RH})$. In both LMMs and hierarchical regression, when conducting analyses across the entire age range ("all" group - both), we used the logarithm of age $\left(\log _{10}\right.$ [age]) as a covariate to account for the possible nonlinear age dependence in childhood, adolescence, and adulthood. 
Table 1. Characteristics of the study participants

\begin{tabular}{lcccccc}
\hline & \multicolumn{2}{l}{ Children and adolescents } & & \multicolumn{2}{l}{ Adults } & \\
\cline { 2 - 3 } \cline { 5 - 6 } & TD & ASD & & TD & ASD \\
\hline Participants, $n$ & 36 & 58 & & 19 & 19 \\
Male:female ratio & $28: 8$ & $51: 7$ & & $19: 0$ & $19: 0$ \\
Handedness, right:left & $31: 5$ & $53: 5$ & & $17: 2$ & $17: 2$ \\
Age, years & $9.21 \pm 1.6$ & $10.07 \pm 2.38$ & & $26.97 \pm 1.29$ & $23.80 \pm 6.26$ \\
Social responsiveness scale-2 & $48.34 \pm 14.46$ & $70.10 \pm 15.34$ & & $41.55 \pm 3.28$ & $67.16 \pm 11.73$ \\
Full scale IQ (estimated) & $113.00 \pm 15.59$ & $103.66 \pm 20.79$ & & $113.75 \pm 15.52$ & $108.36 \pm 19.81$ \\
Verbal IQ & $110.22 \pm 14.64$ & $98.54 \pm 19.17$ & & $119.08 \pm 18.34$ & $106.84 \pm 19.05$ \\
Nonverbal IQ & $111.60 \pm 15.32$ & $106.74 \pm 18.37$ & & $105.16 \pm 11.89$ & $107.84 \pm 20.34$ \\
\hline
\end{tabular}

Estimated full scale IQ, verbal IQ, and nonverbal IQ: from the WISC-IV and the WASI-II.

We calculated averaged M50 and M100 latencies across hemisphere and frequency and age to visualize the scatter plot relationships (per group) between latency and age. Bonferroni correction was applied for multiple comparisons. All statistical analyses were performed with SPSS Statistics version 25 (IBM, Armonk, NY, USA).

\section{Results}

\section{Demographics}

One hundred and thirty-two participants (age 6-42 years) were included in the final analyses (children/adolescents; TD, $n=36,9.2 \pm 1.6$ years; ASD; $n=58,10.1 \pm$ 2.4 years, adults; TD, $n=19,27.0 \pm 1.3$ years, ASD, $n=$ $19,23.8 \pm 6.3$ years; Table 1$)$.

Children/Adolescents. There was a slight but statistically significant difference between groups in age $(t=$ $-1.98, p<0.05$ ), with the children with ASD being $\sim 10$ months older than the TD comparison group. Age was thus used as a covariate in all analyses. There were also, as expected, group differences in GAI/FSIQ $(t=2.32, p<$ $0.05)$, VIQ $(t=3.22, p<0.05)$, and SRS-2 $(t=-7.23, p<$ $0.05)$, but not in NVIQ $(t=1.38, p>0.05$; Table 1$)$.

Adults. There were statistically significant main effects of group on SRS-2 $(t=-6.36, p<0.05)$, but not on GAI/ FSIQ $(t=0.79, p>0.05), \operatorname{VIQ}(t=1.76, p>0.05)$, or NVIQ $(t=-0.41, p>0.05)$. There was no group difference in age $(t=0.78, p>0.05)$.

\section{M50 and M100 Latencies}

Example waveforms from children and adults with TD and ASD are shown in Figure 1.

Children/Adolescents. An LMM with fixed effects of group, frequency, and hemisphere on M50 latency with age as a covariate showed a significant effect of group
$\left(\mathrm{TD}=65.03 \pm 2.12 \mathrm{~ms}, \mathrm{ASD}=71.24 \pm 1.13 \mathrm{~ms}, F_{1,75.00}=\right.$ $6.65, p<0.05)$ (Fig. 2) and frequency $(200 \mathrm{~Hz} ; 67.08 \pm 1.33$ $\mathrm{ms}, 300 \mathrm{~Hz} ; 68.67 \pm 1.33 \mathrm{~ms}, 500 \mathrm{~Hz} ; 69.09 \pm 1.33 \mathrm{~ms}$, $\left.1,000 \mathrm{~Hz} ; 67.90 \pm 1.33 \mathrm{~ms}, F_{3,525}=2.68, p=0.046\right)$ with no effect of hemisphere $(\mathrm{LH}=67.93 \pm 1.28 \mathrm{~ms}, \mathrm{RH}=$ $\left.68.44 \mathrm{~ms} \pm 1.28, F_{1,525}=0.89, p=0.347\right)$ and no interactions between factors $(p>0.05)$. Despite the overall statistical significance of the effect of frequency, none of the pairwise comparisons between frequencies achieved individual significance. A similar LMM performed for M100 latency showed a significant effect of group (TD = $108.33 \pm 2.4 \mathrm{~ms}, \mathrm{ASD}=118.09 \pm 1.2 \mathrm{~ms}, F_{1,75.56}=13.13$, $p<0.001)$ and frequency $(200 \mathrm{~Hz} ; 111.26 \pm 1.46 \mathrm{~ms}, 300$ $\mathrm{Hz} ; 112.97 \pm 1.48 \mathrm{~ms}, 500 \mathrm{~Hz} ; 114.89 \pm 1.46 \mathrm{~ms}, 1,000 \mathrm{~Hz}$; $\left.113.73 \pm 1.42 \mathrm{~ms} ; F_{3,506.89}=5.21, p<0.05\right)$ with no effect of hemisphere $(\mathrm{LH}=112.52 \pm 1.33 \mathrm{~ms}, \mathrm{RH}=114.08$ $\left.\mathrm{ms} \pm 1.34, F_{1,506.65}=5.70, p<0.05\right)$ and no interactions between factors $(p>0.05)$. Post hoc $t$ tests show only the contrast between M100 latency to $200-$ vs. $500-\mathrm{Hz}$ tones achieved significance.

Adults. An LMM with fixed effects of group, frequency, and hemisphere on M50 latency with age showed a significant effect of group $(\mathrm{TD}=46.84 \pm 1.62 \mathrm{~ms}$, ASD $=$ $\left.52.52 \pm 1.83 \mathrm{~ms}, F_{1,31.30}=5.44, p<0.05\right)$ but not frequency $(200 \mathrm{~Hz} ; 49.52 \pm 1.33 \mathrm{~ms}, 300 \mathrm{~Hz} ; 48.97 \pm 1.33 \mathrm{~ms}, 500$ $\mathrm{Hz} ; 50.54 \pm 1.33 \mathrm{~ms}, 1,000 \mathrm{~Hz} ; 49.68 \pm 1.33 \mathrm{~ms} . F_{3,212.04}=$ $1.09, p=0.353)$ or hemisphere $(\mathrm{LH}=49.63 \pm 1.26 \mathrm{~ms}$, $\left.\mathrm{RH}=49.73 \pm 1.26 \mathrm{~ms}, F_{1,212.04}=0.024, p=0.877\right)$ and no interactions between factors $(p>0.05)$.

For M100 latency, the LMM showed significant effect of both group $[\mathrm{TD}=102.56 \pm 2.16 \mathrm{~ms}, \mathrm{ASD}=111.44 \pm$ $\left.2.33 \mathrm{~ms}, F_{1,32.00}=7.80, p<0.01\right]$ and frequency $(200 \mathrm{~Hz}$; $110.36 \pm 1.84 \mathrm{~ms}, 300 \mathrm{~Hz} ; 109.98 \pm 1.84 \mathrm{~ms}, 500 \mathrm{~Hz}$; $106.02 \pm 1.84 \mathrm{~ms}, 1,000 \mathrm{~Hz} ; 101.64 \pm 1.84 \mathrm{~ms}, F_{3,223.03}=$ 


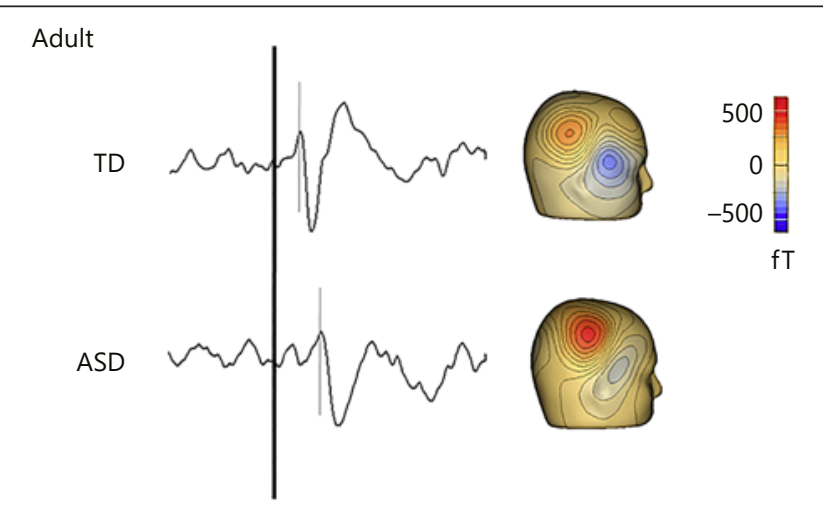

Child

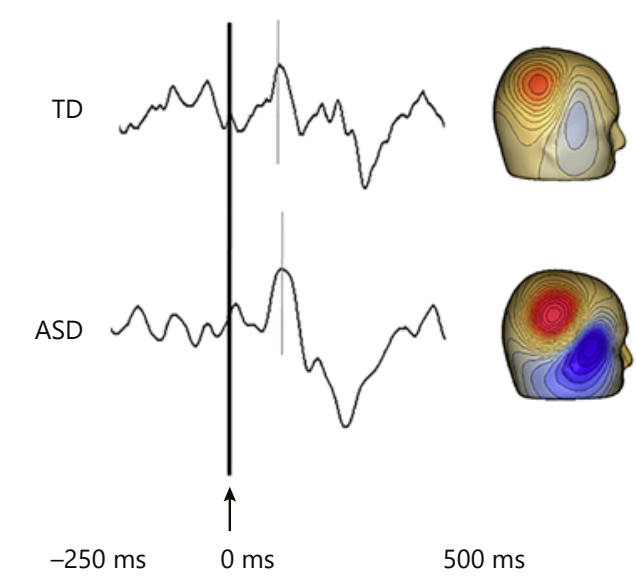

Fig. 1. Source-modeled activity waveform from the right superior temporal gyrus for a representative participant in each group. Black vertical lines on the waveform and arrow indicate stimulus onset (zero ms). Gray lines indicate M50 peaks for the representative TD adult marked at $59 \mathrm{~ms}$ and for the representative ASD adult marked at $79 \mathrm{~ms}$ and TD child marked at $101 \mathrm{~ms}$, for the representative ASD child marked at $117 \mathrm{~ms}$. A clear prolongation of latency is observed in ASD in both child and adult.

$14.57, p<0.001)$ with no effect of hemisphere $(\mathrm{LH}=$ $107.90 \pm 1.68 \mathrm{~ms}, \mathrm{RH}=106.10 \pm 1.68 \mathrm{~ms}, F_{1,223.03}=2.81$, $p=0.095)$ and no interactions between factors $(p>0.05)$. Post hoc $t$ tests showed pairwise M100 latency differences for $200>500 \mathrm{~Hz}, 200>1,000 \mathrm{~Hz}, 300>1,000 \mathrm{~Hz}$, and $500>10,000 \mathrm{~Hz}$.

All (Combined) Group. An LMM with fixed effects of group, frequency, and hemisphere on M50 latency showed a significant effect of group $(\mathrm{TD}=58.19 \pm 1.48$ $\left.\mathrm{ms}, \mathrm{ASD}=66.70 \pm 1.07 \mathrm{~ms}, F_{1,111.06}=21.57, p<0.001\right)$ and frequency $(200 \mathrm{~Hz} ; 61.60 \pm 0.96 \mathrm{~ms}, 300 \mathrm{~Hz} ; 62.38 \pm$ $0.96 \mathrm{~ms}, 500 \mathrm{~Hz} ; 63.35 \pm 0.96 \mathrm{~ms}, 1,000 \mathrm{~Hz} ; 62.45 \pm 0.96$ $\left.\mathrm{ms}, F_{3,765.56}=3.88, p<0.05\right)$ on M50 latency with no effect of hemisphere $(\mathrm{LH}=62.23 \pm 0.93 \mathrm{~ms}, \mathrm{RH}=62.66 \pm$ $\left.0.93 \mathrm{~ms}, F_{1,765.565}=1.42, p=0.232\right)$ and no interactions between factors $(p>0.05)$. Post hoc tests showed the direction of frequency dependence of M50 latency to be inconsistent $(200<500 \mathrm{~Hz}$, but $500>1,000 \mathrm{~Hz}$ and with only the pairwise contrast between 200 and $500 \mathrm{~Hz}$ reaching significance). A similar LMM was performed for M100 latency, which showed a significant effect of group (TD = $107.02 \pm 1.48 \mathrm{~ms}, \mathrm{ASD}=116.71 \pm 1.07 \mathrm{~ms}, F_{1,111.34}=$ $28.03, p<0.001)$ and frequency $(200 \mathrm{~Hz} ; 112.09 \pm 1.00 \mathrm{~ms}$, $300 \mathrm{~Hz} ; 112.73 \pm 1.01 \mathrm{~ms}, 500 \mathrm{~Hz} ; 112.28 \pm 1.01 \mathrm{~ms}, 1,000$ $\left.\mathrm{Hz} ; 110.36 \pm 1.01 \mathrm{~ms}, F_{3,757.96}=4.26, p<0.01\right)$ with no effect of hemisphere $(\mathrm{LH}=111.63 \pm 0.94 \mathrm{~ms}, \mathrm{RH}=112.11 \pm$ $\left.0.95 \mathrm{~ms}, F_{1,758.50}=0.92, p=0.337\right)$ and no interactions between factors $(p>0.05)$. Post hoc tests showed the effect of frequency on M100 latency was smaller but consistent in direction with prior reports, with $200>1,000$ $\mathrm{Hz}(p=0.09), 300>1,000 \mathrm{~Hz}(p<0.01)$ and $500>1,000$ $\mathrm{Hz}(p<0.05)$. Importantly, group differences (of 7-13 $\mathrm{ms})$ were maintained at all tone frequencies.

\section{Association between Age, Language Aptitude, and M50/M100 Latency}

Children/Adolescents. When entered after group, hemisphere, and frequency (together $R^{2}=0.072, p<$ $0.001)$, age accounted for significant additional variance in M50 latency $\left(R^{2}=0.093, \Delta R^{2}=0.021, p<0.001\right)$. Social impairments (SRS score) did not account for significant additional variance $\left(R^{2}=0.098, \Delta R^{2}=0.005, p>0.05\right)$, but language aptitude did account for significant additional variance $\left(R^{2}=0.129, \Delta R^{2}=0.032, p<0.001\right)$. When the order of entry for language and social impairment was reversed, language aptitude continued to account for significant additional variance $\left(R^{2}=0.128, \Delta R^{2}=0.035, p<\right.$ 0.001 ), and social impairments did not account for significant additional variance $\left(R^{2}=0.130, \Delta R^{2}=0.002, p>\right.$ $0.05)$, suggesting statistically significant contributions of age and language aptitude (but not social impairment) to M50 latency. When entered after group, hemisphere, and frequency (together $R^{2}=0.112, p>0.05$ ), age did not account for significant additional variance in M100 latency $\left(R^{2}=0.122, \Delta R^{2}=0.000, p>0.05\right)$, but social impairments indeed accounted for significant additional variance $\left(R^{2}=0.142, \Delta R^{2}=0.020, p<0.001\right)$, and language aptitude also accounted for significant additional variance $\left(R^{2}=\right.$ $\left.0.264, \Delta R^{2}=0.121, p<0.001\right)$. When the order of entry for language aptitude and social impairment was reversed, language aptitude continued to account for significant additional variance $\left(R^{2}=0.257, \Delta R^{2}=0.135, p<\right.$ 


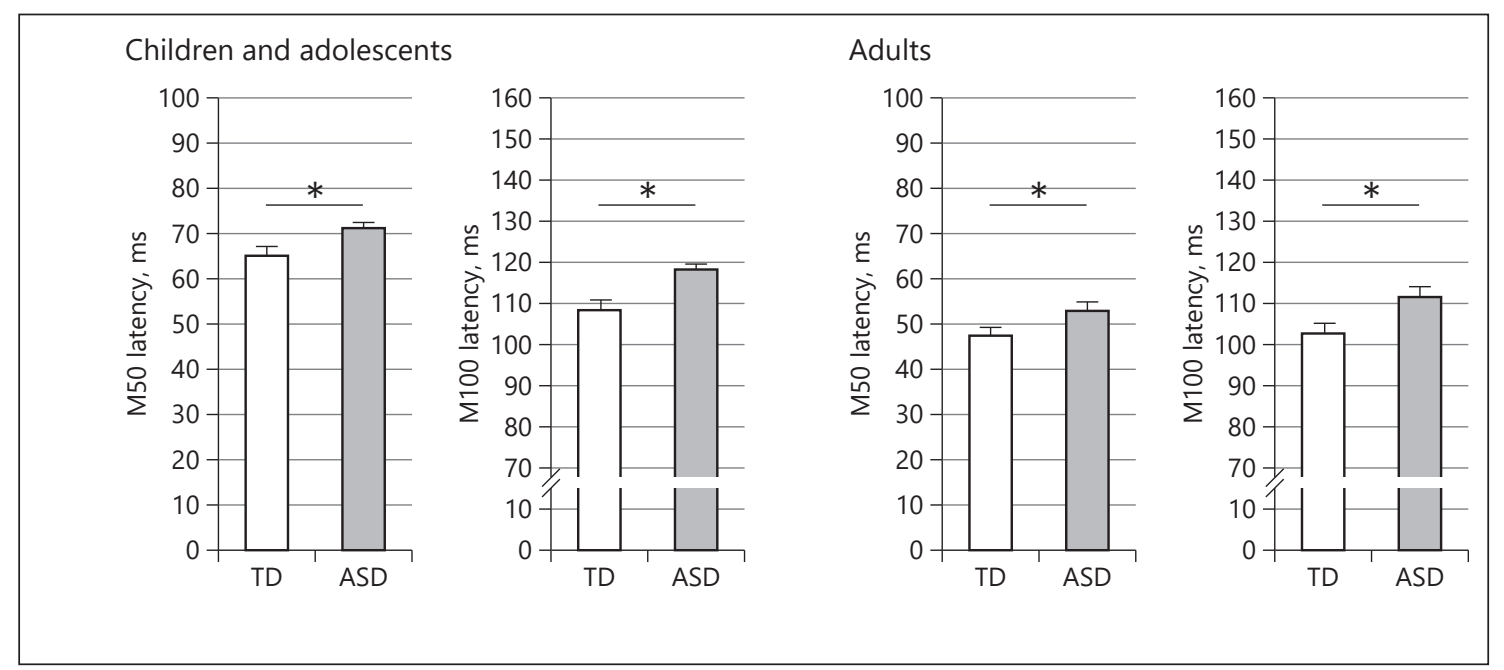

Fig. 2. Estimated marginal mean latencies by group across hemisphere for M50 and M100 latencies. Error bars represent 1 SD of the marginal means. There is a significant main effect of group on M50 and M100 latency $(p<$ $0.05)$ across hemispheres. ASD showed significantly delayed M50 and M100 latencies compared to TD across frequency and hemisphere in both children and adults $(p<0.05)$.

Table 2. Hierarchical regression

\begin{tabular}{|c|c|c|c|}
\hline $\begin{array}{l}\text { M50 latency } \\
\text { Group/hemisphere/frequency }\end{array}$ & $R^{2}=0.086, p<0.001$ & Group/hemisphere/frequency & $R^{2}=0.086, p<0.001$ \\
\hline $\begin{array}{l}\text { Group/hemispheres/frequency + } \\
\text { age }\end{array}$ & $\begin{array}{l}R^{2}=0.354, \Delta R^{2}=0.269 \\
p<0.001\end{array}$ & Group/hemisphere/frequency + age & $\begin{array}{l}R^{2}=0.354, \Delta R^{2}=0.269, \\
\boldsymbol{p}<\mathbf{0 . 0 0 1}\end{array}$ \\
\hline $\begin{array}{l}\text { Group/hemisphere/frequency + } \\
\text { age + SRS-2 }\end{array}$ & $\begin{array}{l}R^{2}=0.358, \Delta R^{2}=0.004 \\
\boldsymbol{p}<\mathbf{0 . 0 5}\end{array}$ & $\begin{array}{l}\text { Group/hemisphere/frequency + age + } \\
\text { VIQ }\end{array}$ & $\begin{array}{l}R^{2}=0.384, \Delta R^{2}=0.030, \\
\boldsymbol{p}<\mathbf{0 . 0 0 1}\end{array}$ \\
\hline $\begin{array}{l}\text { Group/hemisphere/frequency + } \\
\text { age + SRS-2 + VIQ }\end{array}$ & $\begin{array}{l}R^{2}=0.387, \Delta R^{2}=0.029 \\
\boldsymbol{p}<\mathbf{0 . 0 0 1}\end{array}$ & $\begin{array}{l}\text { Group/hemisphere/frequency + age + } \\
\text { VIQ + SRS-2 }\end{array}$ & $\begin{array}{l}R^{2}=0.387, \Delta R^{2}=0.003, \\
\boldsymbol{p}<\mathbf{0 . 0 5}\end{array}$ \\
\hline $\begin{array}{l}\text { M100 latency } \\
\text { Group/hemisphere/frequency }\end{array}$ & $R^{2}=0.114, \boldsymbol{p}<\mathbf{0 . 0 0 1}$ & Group/hemisphere/frequency & $R^{2}=0.114, \boldsymbol{p}<\mathbf{0 . 0 0 1}$ \\
\hline $\begin{array}{l}\text { Group/hemisphere/frequency + } \\
\text { age }\end{array}$ & $\begin{array}{l}R^{2}=0.150, \Delta R^{2}=0.035 \\
\boldsymbol{p}<\mathbf{0 . 0 0 1}\end{array}$ & Group/hemisphere/frequency + age & $\begin{array}{l}R^{2}=0.150, \Delta R^{2}=0.035, \\
\boldsymbol{p}<\mathbf{0 . 0 0 1}\end{array}$ \\
\hline $\begin{array}{l}\text { Group/hemisphere/frequency + } \\
\text { age + SRS-2 }\end{array}$ & $\begin{array}{l}R^{2}=0.152, \Delta R^{2}=0.002 \\
p>0.05\end{array}$ & $\begin{array}{l}\text { Group/hemisphere/frequency + age + } \\
\text { VIQ }\end{array}$ & $\begin{array}{l}R^{2}=0.238, \Delta R^{2}=0.088 \\
\boldsymbol{p}<\mathbf{0 . 0 0 1}\end{array}$ \\
\hline $\begin{array}{l}\text { Group/hemisphere/frequency + } \\
\text { age + SRS-2 + VIQ }\end{array}$ & $\begin{array}{l}R^{2}=0.240, \Delta R^{2}=0.088 \\
\boldsymbol{p}<\mathbf{0 . 0 0 1}\end{array}$ & $\begin{array}{l}\text { Group/hemisphere/frequency + age + } \\
\text { VIQ + SRS-2 }\end{array}$ & $\begin{array}{l}R^{2}=0.240, \Delta R^{2}=0.002, \\
p>0.05\end{array}$ \\
\hline
\end{tabular}

0.001), and social impairments accounted for slight but significant additional variance $\left(R^{2}=0.264, \Delta R^{2}=0.006\right.$, $p<0.05$ ), suggesting statistically significant contributions of language aptitude to M100 latency as well as a moderate influence of social impairment.

Adults. When entered after group, hemisphere, and frequency (together $R^{2}=0.272, p<0.001$ ), age accounted for significant additional variance in M50 latency $\left(R^{2}=\right.$ $\left.0.297, \Delta R^{2}=0.025, p<0.05\right)$, social impairments did not account for significant additional variance $\left(R^{2}=0.298\right.$, $\left.\Delta R^{2}=0.004, p>0.05\right)$, and language aptitude also did not account for significant additional variance $\left(R^{2}=0.301\right.$, $\left.\Delta R^{2}=0.002, p>0.05\right)$. When the order of entry for language and social impairment was reversed, neither lan- 


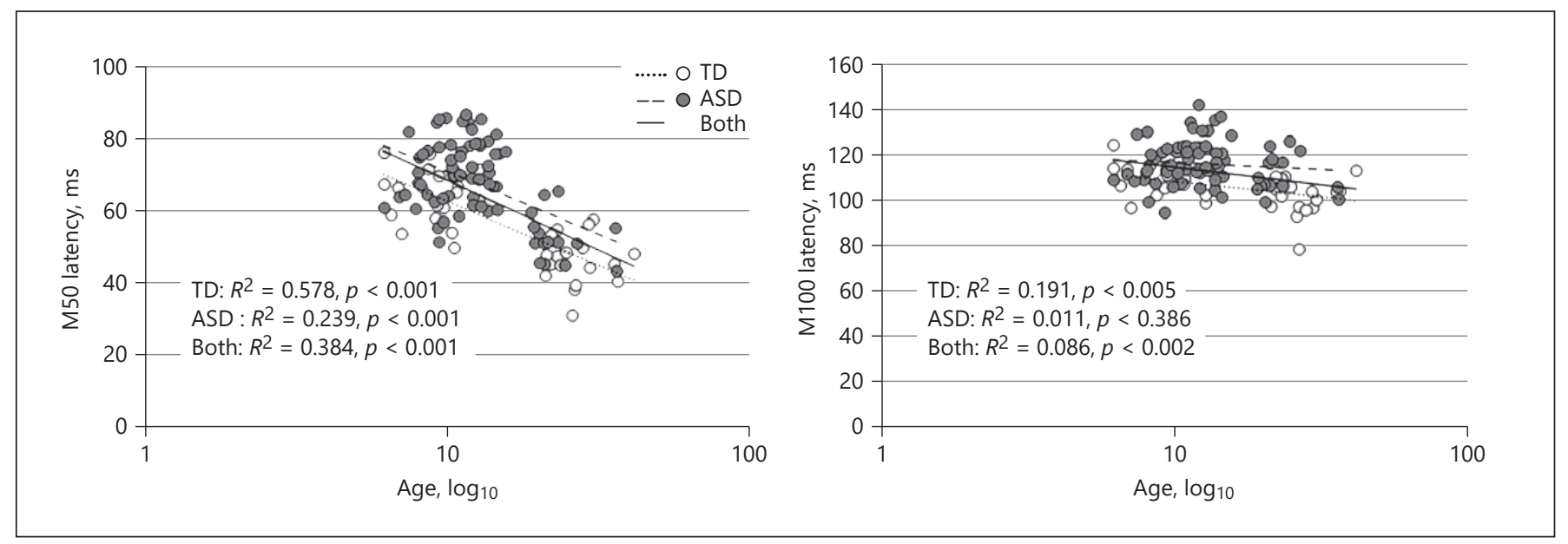

Fig. 3. Scatter plot of M50 and M100 latencies (averaged across hemisphere and frequency) versus $\log _{10}$ (age) shows a statistically significant negative association across groups (M50: $R^{2}=0.384, p<0.001, \mathrm{M} 100: R^{2}=0.086$, $p=0.002)$ and within group M50 and M100 versus $\log _{10}$ (age) associations and their respective $R^{2}$ (M50: TD: $R^{2}=0.578, p<0.001$, ASD: $R^{2}=0.239, p<0.001$; M100: TD: $R^{2}=0.191, p=0.005$, ASD: $\left.R^{2}=0.011, p=0.398\right)$.

guage aptitude $\left(R^{2}=0.295, \Delta R^{2}=0.000, p>0.05\right)$ nor social impairments $\left(R^{2}=0.301, \Delta R^{2}=0.006, p>0.05\right)$ accounted for significant additional variance suggesting statistically significant contributions only of age to M50 latency.

When entered after group, hemisphere, and frequency (together $R^{2}=0.158, p<0.05$ ), age accounted for significant additional variance in M100 latency $\left(R^{2}=0.178\right.$, $\left.\Delta R^{2}=0.019, p<0.05\right)$ but neither social impairments $\left(R^{2}=0.192, \Delta R^{2}=0.014, p>0.05\right)$ nor language aptitude $\left(R^{2}=0.199, \Delta R^{2}=0.007, p>0.05\right)$ accounted for significant additional variance. When the order of entry for language aptitude and social impairment was reversed, language aptitude accounted for slight but significant additional variance $\left(R^{2}=0.194, \Delta R^{2}=0.016, p<0.05\right)$ while social impairments did not account for significant additional variance $\left(R^{2}=0.199, \Delta R^{2}=0.005, p>0.05\right)$, suggesting small but statistically significant contributions of age and language aptitude to M100 latency in adults.

All (Combined) Group. Regarding associations between language aptitude and M50/M100 latency across the life span (Table 2), when entered after group, hemisphere, and frequency (together $R^{2}=0.086, p<0.001$ ), $\log _{10}$ (age) accounted for significant additional variance in M50 latency $\left(R^{2}=0.354, \Delta R^{2}=0.269, p<0.001\right)$, social impairments (SRS score) accounted for slight but significant additional variance $\left(R^{2}=0.358, \Delta R^{2}=0.004, p<\right.$ $0.05)$, and language aptitude also accounted for significant additional variance $\left(R^{2}=0.387, \Delta R^{2}=0.029, p<\right.$ $0.001)$, with higher verbal IQ predicting shorter M50 la-

Delayed Auditory Response in ASD across the Life Span tency and higher SRS score predicting longer M50 latency. When the order of entry for language aptitude and social impairment was reversed, language aptitude accounted for significant additional variance $\left(R^{2}=0.384\right.$, $\left.\Delta R^{2}=0.030, p<0.001\right)$, and social impairments accounted for slight but significant additional variance $\left(R^{2}=\right.$ 0.387, $\left.\Delta R^{2}=0.003, p<0.05\right)$, which suggested statistically significant contributions of age and language aptitude and small contributions of social impairments to M50 latency. When considering the ASD cohort alone, M50 latency was also seen to be negatively associated with language aptitude $\left(R^{2}=0.267, \Delta R^{2}=0.061, p<0.001\right)$.

When entered after group, hemisphere, and frequency (together $R^{2}=0.114, p<0.001$ ), age accounted for significant additional variance in M100 latency $\left(R^{2}=0.150\right.$, $\left.\Delta R^{2}=0.035, p<0.001\right)$, social impairments did not account for significant additional variance $\left(R^{2}=0.152\right.$, $\left.\Delta R^{2}=0.002, p>0.05\right)$, but language aptitude did account for significant additional variance $\left(R^{2}=0.240, \Delta R^{2}=\right.$ $0.088, p<0.001)$. When the order of entry for language aptitude and social impairment was reversed, language aptitude accounted for significant additional variance $\left(R^{2}=0.238, \Delta R^{2}=0.088, p<0.001\right)$, but social impairments did not account for significant additional variance $\left(R^{2}=0.240, \Delta R^{2}=0.002, p>0.05\right)$, which suggests statistically significant contributions of age and language aptitude to M100 latency. When considering the ASD cohort alone, M100 latency was seen to be negatively associated with language aptitude $\left(R^{2}=0.162, \Delta R^{2}=0.125, p<\right.$ $0.001)$. 
A scatter plot of M50 and M100 latency (Fig. 3, averaged across hemisphere and frequency) versus $\log _{10}$ (age) shows a statistically significant negative association across groups (M50: $R^{2}=0.384, p<0.001, \mathrm{M} 100: R^{2}=0.086, p=0.002$ ). Figure 3 also reports the within group M50 and M100 versus $\log _{10}$ (age) associations and their respective $R^{2}$ (M50: TD: $R^{2}=0.578, p<0.001$, ASD: $R^{2}=0.239, p<0.001$; M100: TD: $R^{2}=0.191, p=0.005$, ASD: $\left.R^{2}=0.011, p=0.398\right)$ as well as their slopes (M50: TD $=-35.15 \mathrm{~ms} / \log _{10}$ [year] vs. ASD $=-34.69 \mathrm{~ms} / \log _{10}$ [year] - note $-35 \mathrm{~ms} / \log _{10}$ [year] corresponds to instantaneous rates of M50 shortening of $2.5 \mathrm{~ms} /$ year at age $6,1.5 \mathrm{~ms} /$ year at age 10 , and $0.5 \mathrm{~ms} /$ year at age 30 , for example; M100: TD $=-14.0 \mathrm{~ms} / \log _{10}$ [year] vs. ASD $=-6.1 \mathrm{~ms} / \log _{10}$ [year]; note: $14.0 \mathrm{~ms} / \log _{10}$ [year] corresponds to $0.6 \mathrm{~ms} /$ year at age 10 , while $-6.1 \mathrm{~ms} /$ year corresponds to $0.3 \mathrm{~ms} /$ year at age 10 , for example).

\section{Discussion}

The main finding of this study is that there are delays in the M50 and M100 latency in individuals with ASD compared to TD controls, and these delays persist across the life span with no evidence of either convergence or increase. Across the life span, we observed a significant negative association between M50 latency and age for both groups, but a similar association between M100 latency and age, observed in the TD group, was not seen in the ASD group, which showed little evidence of maturation. Furthermore, delayed M50 and M100 latencies were associated with poorer verbal aptitude as indexed by the VIQ in the whole cohort.

Previous studies have shown delayed M50 or M100 latencies in children or adults with ASD [3-15], along with reported differences in the maturational trajectory [8].

In this study, we report the observation that these latency delays persist into adulthood with no evidence of either convergence or divergence. The origin of the latency delays is still unclear, but has been speculatively attributed to both white matter conduction velocity [17] and speed of synaptic transmission [37] along the auditory pathway. Regarding local synaptic transmission, development of layers (lower III-VI) in the auditory cortex is known to occur between 6 months and 5 years of age [38], with superficial layers (upper layer III and II) continuing to mature until about 12 years [39-41]. The auditory 50 -ms response reflects recurrent activation in layers III and IV, the termination zone of thalamocortical pathways that are almost fully developed by age 6 years, and the 100-ms auditory response reflects activation of upper cortical layers III and II, areas not fully developed until 12 years of age $[42,43]$. In the present study, delayed M50 and M100 latencies were found across the life span (both children/adolescents and adults) and suggest that findings of latency delays in both M50 and M100 perhaps indicate that abnormalities in maturation of the local neural circuits generating these responses persist into adulthood, or that atypical maturation during critical periods of development lead to irrevocable response delays that persist in adults, not normalized by subsequent maturation during later adolescence/adulthood.

An alternative (or adjunct) to the cortical maturation hypothesis is consideration of the thalamus itself and thalamocortical connectivity, which plays a key role in sensory modalities [44] as well as language aptitude [45]. Concerning thalamic abnormalities in individuals with ASD across the life span, there have been PET and fMRI reports suggesting abnormal thalamocortical networks, involved in language processing, in both children and adults $[46,47]$. Thus, abnormalities in thalamocortical networks have been hypothesized to be related to language ability, and those abnormalities might also be evidence in all developmental stages.

Another finding is the association between language aptitude (VIQ) and M50 and M100 latencies. The M50 and M100 responses are primarily generated in the STG [48-50], and it has figured prominently in models of receptive language function and impairment [51]. In an early MEG study, Oram Cardy et al. [6] reported that longer M50 latencies predicted impaired receptive language ability in children and adolescents (aged 7-18 years) across typically developing controls, children with ASD, children with Asperger's syndrome, and children with specific language impairment. The authors suggested basic non-speech auditory processing is specifically linked to oral language functioning, and that latency delays evident at this early stage of auditory perception may specifically contribute to impaired language development. In the present study, associations between M50 and M100 latencies and VIQ were observed across the life span. Of note, VIQ, an index of verbal intelligence or aptitude, used in this study may not be directly compared with measures of language ability, such as those derived from the CELF.

Efficient processing in the auditory cortex is essential for real-time speech-based interaction. Therefore, perhaps it is not surprising that these delays exhibit an association with poor language aptitude across the life span.

While the use of an electrophysiological signature is unlikely to be of value in either the adolescent or adult population as a diagnostic biomarker, the above-reported 
delays might still be of value in suggesting substrates for intervention and perhaps as signals of efficacy of such interventions (whether behavioral or pharmacological). Indeed, patients might be stratified according to their delay and management options tailored to suit their particular biological profile.

Importantly, it appears that such signatures persist into adulthood and thus may offer similar stratification and patient management value in young adults as well as children, potentially suggesting a more widely open therapeutic window.

Limitations of this study include the lack of infant participants, sufficient female participants to statistically consider effects of sex, as well as not extending the age range towards the middle-aged and aging population. It remains of great interest to explore associations between sensory processing signatures such as the M50 and M100 latency and measures of natural aging and cognitive decline. It is possible that identification of similar hallmarks might even allow for shared interventional strategies. Another study limitation is that brain imaging measures derived from other modalities were not assessed (e.g., cortical myelin content, diffusion tensor imaging, and/or GABA magnetic resonance spectroscopy). Further studies are needed combining other modalities to develop appropriate objective markers associated with language ability in individuals with ASD across the life span. Previous reports have noted effects of frequency on M100 latency with lower frequencies having longer latencies [52]. No such association has been reported for M50 latencies. Considering the combined cohort, we indeed observed a similarly directioned effect for M100 latency, although of rather small magnitude. For M50, we also observed a significant effect of frequency, but since the post hoc $t$ tests were of inconsistent directionality and significance, and since the magnitude of the effect was very small, we interpret this as rather statistical noise. Separately considering children and adults, we were only able to resolve clear M100 latency dependence on stimulus frequency in the adult cohort, perhaps suggesting the lack of robustness of this observation in the developing brain as well as suggesting that such frequency encoding or representation may be unique to the later M100 component and not evident in the earlier M50 component. It is possible that methodological differences from previous studies, sample sizes, or other sources of variance obscure a resolvable latency dependence in children. Nonetheless, tone frequency is incorporated into all statistical analyses presented.

In summary, delayed M50 and M100 auditory evoked component latencies were observed in ASD, consistent with prior reports. These delays persisted into adulthood, with no evidence of convergence (or divergence). These latency delays were associated with poorer language performance (assessed by VIQ), pointing to a neural correlate of impaired behavioral performance in ASD, across the life span.

\section{Acknowledgments}

All authors gratefully acknowledge all participants and their families. Dr. Timothy P.L. Roberts thanks the Oberkircher Family for the Oberkircher Family Chair in Pediatric Radiology. All authors also thank Rachel Golembski, Peter Lam, Erin Huppman, Na'Keisha Robinson, and the MEG lab team (CHOP Department of Radiology).

\section{Statement of Ethics}

The study was approved by the CHOP Internal Review Board. Written informed consent and assent (when age appropriate) was obtained from all participants or participating families, in accordance with the principles of the Declaration of Helsinki.

\section{Disclosure Statement}

Dr. Timothy P. L. Roberts declares consulting/advisory board relationships with Prism Clinical Imaging, CTF, Ricoh, Spago Nanomedical, Avexis Inc., and Acadia Pharmaceuticals. Additionally, he discloses intellectual property related to MEG as a biomarker for pharmaceutical therapy, under licensing negotiation. All other authors have no disclosures.

\section{Funding Sources}

This study was supported in part by NIH R01-DC008871 (T.P.L.R.), a maturational human biology grant from the Institute for Translational Medicine and Therapeutics, University of Pennsylvania (UL1-RR024134, T.P.L.R./E.S.B.), as well as the institutional Intellectual and Developmental Disabilities Research Center (U54-HD086984, Project PI: T.P.L.R.) and NIH R01-HD073258 (D.E.).

\section{Author Contributions}

T.P.L.R., E.S.B., and D.E.: study design. T.C.: recruitment. L. Blaskey, E.S.K., and M. Kim: neuropsychology. J.D., M.D., M. Ku, M. Kim, J.M., and T.C.: data acquisition and preprocessing. L. Bloy and S.L.: computer code generation. M. Ku, J.M., J.S., and Y.C.: data processing. J.M., T.P.L.R., L. Bloy, and J.I.B.: statistical analysis. J.M. and T.P.L.R.: manuscript first drafting. L. Blaskey, L. Bloy, Y.C., J.I.B., J.S., E.S.B., D.E., and M. Kim: manuscript editing. All authors: manuscript review. 


\section{References}

1 Kenny L, Hattersley C, Molins B, Buckley C, Povey C, Pellicano E. Which terms should be used to describe autism? Perspectives from the UK autism community. Autism. 2016 May;20(4):442-62.

2 American Psychiatric Association. Diagnostic and Statistical Manual of Mental Disorders. 5th ed. Washington (DC): American Psychiatric Publishing, Inc.; 2013.

3 Gage NM, Siegel B, Roberts TP. Cortical auditory system maturational abnormalities in children with autism disorder: an MEG investigation. Brain Res Dev Brain Res. 2003 Sep; 144(2):201-9.

4 Gage NM, Siegel B, Callen M, Roberts TP. Cortical sound processing in children with autism disorder: an MEG investigation. Neuroreport. 2003 Nov;14(16):2047-51.

5 Oram Cardy JE, Ferrari P, Flagg EJ, Roberts W, Roberts TP. Prominence of M50 auditory evoked response over M100 in childhood and autism. Neuroreport. 2004 Aug;15(12):186770.

6 Oram Cardy JE, Flagg EJ, Roberts W, Roberts TP. Auditory evoked fields predict language ability and impairment in children. Int J Psychophysiol. 2008 May;68(2):170-5.

7 Roberts TP, Khan SY, Rey M, Monroe JF, Cannon K, Blaskey L, et al. MEG detection of delayed auditory evoked responses in autism spectrum disorders: towards an imaging biomarker for autism. Autism Res. 2010 Feb; 3(1):8-18

8 Roberts TP, Lanza MR, Dell J, Qasmieh S, Hines K, Blaskey L, et al. Maturational differences in thalamocortical white matter microstructure and auditory evoked response latencies in autism spectrum disorders. Brain Res. 2013 Nov; 1537:79-85.

9 Roberts TPL, Matsuzaki J, Blaskey L, Bloy L, Edgar JC, Kim M, et al. Delayed M50/M100 evoked response component latency in minimally verbal/nonverbal children who have autism spectrum disorder. Mol Autism. 2019 Aug; 10:34.

10 Matsuzaki J, Kagitani-Shimono K, Goto T, Sanefuji W, Yamamoto T, Sakai S, et al. Differential responses of primary auditory cortex in autistic spectrum disorder with auditory hypersensitivity. Neuroreport. 2012 Jan; 23(2):113-8.

11 Yoshimura Y, Kikuchi M, Shitamichi K, Ueno S, Munesue T, Ono Y, et al. Atypical brain lateralisation in the auditory cortex and language performance in 3- to 7-year-old children with high-functioning autism spectrum disorder: a child-customised magnetoencephalography (MEG) study. Mol Autism. 2013 Oct;4(1):38.

12 Edgar JC, Fisk Iv CL, Berman JI, Chudnovskaya D, Liu S, Pandey J, et al. Auditory encoding abnormalities in children with autism spectrum disorder suggest delayed development of auditory cortex. Mol Autism. 2015 Dec;6:69.
13 Port RG, Anwar AR, Ku M, Carlson GC, Siegel SJ, Roberts TP. Prospective MEG biomarkers in ASD: pre-clinical evidence and clinical promise of electrophysiological signatures. Yale J Biol Med. 2015 Mar;88(1):25-36.

14 Stephen JM, Hill DE, Peters A, Flynn L, Zhang T, Okada Y. Development of Auditory Evoked Responses in Normally Developing Preschool Children and Children with Autism Spectrum Disorder. Dev Neurosci. 2017;39(5):430-41.

15 Demopoulos C, Hopkins J, Kopald BE, Paulson K, Doyle L, Andrews WE, et al. Deficits in auditory processing contribute to impairments in vocal affect recognition in autism spectrum disorders: A MEG study. Neuropsychology. 2015 Nov;29(6):895-908.

16 Paetau R, Ahonen A, Salonen O, Sams M. Auditory evoked magnetic fields to tones and pseudowords in healthy children and adults. J Clin Neurophysiol. 1995 Mar;12(2):177-85.

17 Roberts TP, Khan SY, Blaskey L, Dell J, Levy SE, Zarnow DM, et al. Developmental correlation of diffusion anisotropy with auditoryevoked response. Neuroreport. 2009 Dec; 20(18):1586-91.

18 Lord C, Risi S, Lambrecht L, Cook EH Jr, Leventhal BL, DiLavore PC, et al. The autism diagnostic observation schedule-generic: a standard measure of social and communication deficits associated with the spectrum of autism. J Autism Dev Disord. 2000 Jun;30(3):205-23.

19 Lord C, Rutter M, DiLavore PC, Risi S, Gotham K, Bishop SL. Autism Diagnostic Observation Schedule. 2nd ed. Torrance (CA): Western Psychological Services; 2012.

20 Rutter M, Bailey A, Lord C. The Social Communication Questionnaire: Manual. Los Angeles (CA): Western Psychological Services; 2003.

21 Constantino JN, Davis SA, Todd RD, Schindler MK, Gross MM, Brophy SL, et al. Validation of a brief quantitative measure of autistic traits: comparison of the social responsiveness scale with the autism diagnostic interview-revised. J Autism Dev Disord. 2003 Aug;33(4):427-33.

22 Constantino JN, Gruber CP. Social Responsiveness Scale. 2nd ed. Los Angeles (CA): Western Psychological Services; 2012.

23 Lord C, Rutter M, Le Couteur A. Autism Diagnostic Interview-Revised: a revised version of a diagnostic interview for caregivers of individuals with possible pervasive developmental disorders. J Autism Dev Disord. 1994 Oct;24(5):659-85.

24 Wechsler D. Wechsler Intelligence Scale for children. 4th ed. San Antonio (TX): Pearson; 2003.

25 Hurley RS, Losh M, Parlier M, Reznick JS, Piven J. The broad autism phenotype questionnaire. J Autism Dev Disord. 2007 Oct; 37(9):1679-90.

26 Wechsler D. Wechsler Abbreviated Scale of Intelligence. 2nd ed. San Antonio (TX): NCS Pearson; 2011.
27 Semel E, Wiig EH, Secord W. Clinical Evaluation of Language Fundamentals (4th ed.). San Antonio: The Psychological Corporation; 2003.

28 Dunn DM, Dunn LM. Peabody Picture Vocabulary Test. 4th ed., Minneapolis: NCS Pearson, Inc. 2007.

29 Williams KT. Expressive Vocabulary Test. 2nd ed. Minneapolis (MN): NCS Pearson, Inc.; 2007.

30 Scherg M, von Cramon D. A new interpretation of the generators of BAEP waves I-V: results of a spatio-temporal dipole model. Electroencephalogr Clin Neurophysiol. 1985 Jul; 62(4):290-9.

31 Scherg M. Fundamentals of dipole source potential analysis. Adv audiol. 6th ed. Basel, Switzerland: Karger; 1990. pp. 40-69.

32 Scherg M, Berg P. New concepts of brain source imaging and localization. Electroencephalogr Clin Neurophysiol Suppl. 1996;46: 127-37.

33 Lins OG, Picton TW, Berg P, Scherg M. Ocular artifacts in recording EEGs and event-related potentials. II: source dipoles and source components. Brain Topogr. 1993;6(1):65-78.

34 Berg P, Scherg M. A multiple source approach to the correction of eye artifacts. Electroencephalogr Clin Neurophysiol. 1994 Mar; 90(3):229-41.

35 Scherg M, Ebersole JS. Brain source imaging of focal and multifocal epileptiform EEG activity. Neurophysiol Clin. 1994 Jan;24(1):5160.

36 Scherg M, Ille N, Bornfleth H, Berg P. Advanced tools for digital EEG review: virtual source montages, whole-head mapping, correlation, and phase analysis. J Clin Neurophysiol. 2002 Apr;19(2):91-112.

37 Port RG, Edgar JC, Ku M, Bloy L, Murray R, Blaskey L, et al. Maturation of auditory neural processes in autism spectrum disorder - A longitudinal MEG study. Neuroimage Clin. 2016 Apr;11:566-77.

38 Ponton CW, Moore JK, Eggermont JJ. Prolonged deafness limits auditory system developmental plasticity: evidence from an evoked potentials study in children with cochlear implants. Scand Audiol Suppl. 1999; 51:13-22.

39 Moore JK, Guan YL. Cytoarchitectural and axonal maturation in human auditory cortex. J Assoc Res Otolaryngol. 2001 Dec;2(4):297311.

40 Moore JK, Linthicum FH Jr. The human auditory system: a timeline of development. Int J Audiol. 2007 Sep;46(9):460-78.

41 Harris KD, Shepherd GM. The neocortical circuit: themes and variations. Nat Neurosci. 2015 Feb;18(2):170-81.

42 Ponton CW, Vasama JP, Tremblay K, Khosla D, Kwong B, Don M. Plasticity in the adult human central auditory system: evidence from late-onset profound unilateral deafness. Hear Res. 2001 Apr;154(1-2):32-44. 
43 Ponton C, Eggermont JJ, Khosla D, Kwong B, Don M. Maturation of human central auditory system activity: separating auditory evoked potentials by dipole source modeling. Clin Neurophysiol. 2002 Mar;113(3):407-20.

44 Green SA, Hernandez L, Bookheimer SY, Dapretto M. Reduced modulation of thalamocortical connectivity during exposure to sensory stimuli in ASD. Autism Res. 2017 May; 10(5):801-9.

45 Bohsali AA, Triplett W, Sudhyadhom A, Gullett JM, McGregor K, FitzGerald DB, et al. Broca's area - thalamic connectivity. Brain Lang. 2015 Feb;141:80-8.
46 Müller RA, Chugani DC, Behen ME, Rothermel RD, Muzik O, Chakraborty PK, et al. Impairment of dentato-thalamo-cortical pathway in autistic men: language activation data from positron emission tomography. Neurosci Lett. 1998 Mar;245(1):1-4.

47 Linke AC, Jao Keehn RJ, Pueschel EB, Fishman I, Müller RA. Children with ASD show links between aberrant sound processing, social symptoms, and atypical auditory interhemispheric and thalamocortical functional connectivity. Dev Cogn Neurosci. 2018 Jan; 29:117-26.

48 Mäkelä JP, Hämäläinen M, Hari R, McEvoy L. Whole-head mapping of middle-latency auditory evoked magnetic fields. Electroencephalogr Clin Neurophysiol. 1994 Sep;92(5):41421.
49 Pelizzone M, Hari R, Mäkelä JP, Huttunen J, Ahlfors S, Hämäläinen M. Cortical origin of middle-latency auditory evoked responses in man. Neurosci Lett. 1987 Dec;82(3):303-7.

50 Yoshiura T, Ueno S, Iramina K, Masuda K. Human middle latency auditory evoked magnetic fields. Brain Topogr. 1996;8(3):291-6.

51 Gernsbacher MA, Kaschak MP. Neuroimaging studies of language production and comprehension. Annu Rev Psychol. 2003;54(1): 91-114.

52 Roberts TP, Poeppel D. Latency of auditory evoked M100 as a function of tone frequency. Neuroreport. 1996 Apr;7(6):1138-40. 